\title{
Effects of High- and Low-Fat Meals on the Pharmacokinetics of Ozanimod, a Novel Sphingosine- I-Phosphate Receptor Modulator
}

Clinical Pharmacology in Drug Development $2018,7(6) 634-640$ (c) 2017, The Authors. Clinical

Pharmacology in Drug Development Published by Wiley Periodicals, Inc. on behalf of The American College of

Clinical Pharmacology

DOI: $10.1002 /$ cpdd.409

\author{
Jonathan Q. Tran', Jeffrey P. Hartung ${ }^{2}$, Cindy-Ann Tompkins ${ }^{3}$, and Paul A. Frohna ${ }^{4}$
}

\begin{abstract}
Ozanimod (RPCI063) is an oral selective modulator of the sphingosine-I-phosphate I and 5 receptors under development for the treatment of relapsing multiple sclerosis and inflammatory bowel disease. The effects of high-fat and low-fat meals on the pharmacokinetics (PK) of a single oral dose of ozanimod were evaluated in 24 healthy volunteers in a randomized, open-label crossover trial. Each subject received a I-mg dose of ozanimod hydrochloride under 3 meal conditions (fasted, high-fat, and low-fat), each separated by 7 days. Mean plasma concentration-time profiles for ozanimod and its active metabolites (RPI0I988 [major], RPI0I075 [minor]) were similar under all 3 conditions. Moreover, all PK parameters for ozanimod, RPI01988, and RPI0I075 were similar under the 3 meal conditions. The $90 \%$ confidence intervals $(\mathrm{Cls})$ for the ratios of geometric least-squares mean (fed/fasted) were within the equivalence limits of 0.80 to 1.25 for area under the concentration-time curve from time 0 to infinity $\left(\mathrm{AUC}_{0-\infty}\right)$ and maximum plasma concentration $\left(\mathrm{C}_{\max }\right)$ for ozanimod, RPI01988, and RPI01075, except for the high-fat effect on RPI0I075 $\mathrm{C}_{\max }(90 \% \mathrm{Cl}, 0.76-0.88)$. Given this lack of a food effect on the exposure of ozanimod and its active metabolites, ozanimod can be taken without regard to meals.
\end{abstract}

\section{Keywords}

ozanimod, pharmacokinetics, food effects, clinical trial, bioavailability

Chronic immunoinflammatory disorders such as multiple sclerosis (MS) and inflammatory bowel disease (IBD) are commonly treated with immune modulators that target aberrant immune responses. ${ }^{1,2}$ However, currently available agents for these disorders, whether they are injectable or oral, are associated with limitations related to safety/tolerability and convenience/adherence. ${ }^{1,3,4}$ Thus, there is a medical need for convenient, safe, and well-tolerated therapeutic alternatives.

Sphingosine-1-phosphate (S1P) is an active phospholipid that binds to a family of 5 different G-proteincoupled receptor subtypes $\left(\mathrm{S}_{1} \mathrm{P}_{1-5 \mathrm{R}}\right)$. $\mathrm{S} 1 \mathrm{P}$ and its receptors regulate a variety of processes in the immune, cardiovascular, pulmonary, and nervous systems. ${ }^{5,6}$ $\mathrm{S} 1 \mathrm{P}$ receptors are differentially expressed on a wide variety of cells. $\mathrm{S}_{1} \mathrm{P}_{1 \mathrm{R}}, \mathrm{S}_{\mathrm{P}} \mathrm{P}_{2 \mathrm{R}}$, and $\mathrm{S} 1 \mathrm{P}_{3 \mathrm{R}}$ are ubiquitously present in the immune, cardiovascular, and central nervous systems. Expression of $\mathrm{S}_{1} \mathrm{P}_{4 \mathrm{R}}$ is primarily on lymphocytic and hematopoietic cells, and $\mathrm{S}_{1} \mathrm{P}_{5 \mathrm{R}}$ expression is restricted to the spleen (on natural killer cells) and the central nervous system (on oligodendrocytes). ${ }^{7}$

Ozanimod (RPC1063) is an oral selective modulator of S1P receptors with improved selectivity for $\mathrm{S}_{1} \mathrm{P}_{1 \mathrm{R}}$ and $\mathrm{S}_{1} \mathrm{P}_{5 \mathrm{R}}$. Based on favorable disease-amelioration activity in animal models of MS and IBD, ozanimod is currently in clinical development for the treatment of relapsing MS (RMS), moderate to severe ulcerative colitis (UC), and Crohn's disease. ${ }^{8,9}$ Preclinical $\left[{ }^{35} \mathrm{~S}\right]$ GTP $\gamma$ S binding assays established that ozanimod has potent agonist activity of $\mathrm{S}_{1} \mathrm{P}_{1 \mathrm{R}}$ and $\mathrm{S}_{1} \mathrm{P}_{5 \mathrm{R}}$, with mean half-maximal effective concentration $\left(\mathrm{EC}_{50}\right)$ of 0.41 $\pm 0.16 \mathrm{nM}$ and $11 \pm 4.3 \mathrm{nM}$ for $\mathrm{S}_{1 \mathrm{P}} \mathrm{P}_{1 \mathrm{R}}$ and $\mathrm{S}_{\mathrm{P}} \mathrm{P}_{5 \mathrm{R}}$,

\footnotetext{
'Receptos, a wholly owned subsidiary of Celgene, San Diego, CA, USA ${ }^{2}$ JPH Clinical Development, Inc, San Diego, CA, USA

${ }^{3}$ San Diego, CA, USA

${ }^{4}$ Bioniz Therapeutics, Inc., Irvine, CA, USA
}

This is an open access article under the terms of the Creative Commons Attribution-NonCommercial License, which permits use, distribution and reproduction in any medium, provided the original work is properly cited and is not used for commercial purposes.

Submitted for publication I5 May 2017; accepted 20 September 2017.

Corresponding Author:

Jonathan Q. Tran, Executive Director, Clinical Pharmacology Receptos, a wholly owned subsidiary of Celgene Corporation, 3033 Science Park Rd., Suite 300, San Diego, CA 92121

(e mail:jtran@celgene.com) 
respectively. ${ }^{9}$ Two active metabolites, RP101988 (major) and RP101075 (minor), with similar in vitro S1P selectivity as the parent were identified in preclinical species (data on file).

The safety, pharmacokinetics (PK), and pharmacodynamics of ozanimod hydrochloride $(\mathrm{HCl})$ in single oral doses (up to $3 \mathrm{mg}$ ) and multiple oral doses (up to $2 \mathrm{mg}$ once daily) were characterized in a phase 1 study of healthy volunteers. ${ }^{10}$ Following oral administration under fasting conditions, the median time to maximum concentration $\left(\mathrm{T}_{\max }\right)$ was approximately 8 hours. ${ }^{10}$ Ozanimod exhibited linear PK, with doseproportional increases in exposure and low to moderate intersubject variability (\% coefficient of variation, $17 \%$ $32 \%)$, high apparent volume of distribution $(81.9 \mathrm{~L} / \mathrm{kg})$, moderate apparent oral clearance $(233 \mathrm{~L} / \mathrm{h})$, and elimination half-life of approximately 17 hours. ${ }^{10}$ At steady state, the metabolite-to-parent area under the drug concentration-time curve (AUC) ratio for RP101988 and RP101075 is approximately 1.3 and 0.15 , respectively (data on file). Food can affect the PK of drugs in a number of ways, including either decreasing or increasing the rate and/or extent of absorption..$^{11}$ Meal timing, size, and composition (eg, fat, protein, fiber) are factors that influence meal-related changes in the bioavailability of drugs. ${ }^{11}$ Food-induced changes in PK are particularly important for drugs that have a narrow therapeutic index, because small changes in exposure can have clinically meaningful effects on safety and/or efficacy outcomes. ${ }^{12}$

The objective of this study was to characterize the effects of high-fat and low-fat meals on the PK and safety of a single oral dose of ozanimod in healthy adult subjects. Guidelines from the US Food and Drug Administration (FDA) recommend that studies be performed under fasted and fed conditions, with the meal consisting of a high-fat content ( $50 \%$ ) to maximize the potential for an observable food effect. ${ }^{13}$ The low-fat meal was included in addition to the regulatory high-fat meal to provide clinical recommendations across a variety of meal compositions in the event the study results show a clinically meaningful effect from the high-fat meal.

\section{Methods}

The study was reviewed and approved by an institutional review board (IntegReview, Austin, Texas) and was designed and conducted in accordance with the ethical principles of Good Clinical Practice and the Declaration of Helsinki. All subjects provided written informed consent.

\section{Study Design}

This study was a phase 1 randomized, open-label, 3-period, 6-sequence crossover trial conducted at ICON
Early Phase Services, LLC (San Antonio, Texas). Healthy adult subjects were screened for participation within 28 days prior to administration of the first dose of ozanimod. Screening procedures included collection of demographic data, recording of medical/surgical history and vital signs, a complete physical examination, a 12-lead electrocardiogram (ECG), and laboratory evaluations (serology, hematology, chemistry, urinalysis, pregnancy testing, drug and cotinine screening).

Twenty-four subjects were enrolled to receive a single 1-mg oral dose of ozanimod $\mathrm{HCl}$ under 3 different conditions in 3 treatment periods, each separated by a washout period of 7 days. The 3 conditions were fasted (treatment A), with a standard FDA high-fat breakfast (treatment B), and with a low-fat breakfast (treatment C). Eligible subjects were randomized (1:1:1) on the day before their first treatment period to 1 of 6 treatment sequences $(\mathrm{ABC}, \mathrm{ACB}, \mathrm{BAC}, \mathrm{BCA}, \mathrm{CAB}$, or $\mathrm{CBA})$. At the beginning of all 3 treatment periods, subjects were admitted to the clinical research unit (CRU) 1 day before dosing and remained in the CRU, under medical supervision, for 4 days after dosing to complete the study evaluations. A follow-up visit was conducted 7 days after administration of the last dose of ozanimod, which included safety laboratory tests, a complete physical examination, a 12-lead ECG, vital signs, and recording of adverse events (AEs).

\section{Dosing}

Ozanimod $\mathrm{HCl}$ was administered orally as a 1-mg capsule with $240 \mathrm{~mL}$ of noncarbonated room-temperature water at the same time each day under the following 3 conditions according to their assigned treatment sequence:

- Treatment A (fasted): Ozanimod was administered following an overnight fast of at least 10 hours. No food was allowed for at least 4 hours postdose. Water was allowed, as desired, except for 1 hour before and after dosing.

- Treatment B (with a high-fat breakfast): Following an overnight fast of at least 10 hours, subjects started the breakfast 30 minutes prior to ozanimod administration. The highfat breakfast contained 900 to $1000 \mathrm{kcal}$, consisting of protein $(\sim 150 \mathrm{kcal})$, carbohydrate $(\sim 250-360 \mathrm{kcal})$, and fat $(\sim 500-600 \mathrm{kcal})$. Subjects consumed the complete breakfast in 30 minutes or less. No food was allowed for at least 4 hours postdose. Water was allowed, as desired, except for 1 hour before and after dosing.

- Treatment C (with a low-fat breakfast): Following an overnight fast of at least 10 hours, subjects started the breakfast 30 minutes prior to 
ozanimod administration. The low-fat breakfast contained 270 to $340 \mathrm{kcal}$, including protein ( $\sim 40 \mathrm{kcal})$, carbohydrate $(\sim 250 \mathrm{kcal})$, and fat ( $\sim 19-46 \mathrm{kcal})$. Subjects consumed the complete breakfast in 30 minutes or less. No food was allowed for at least 4 hours postdose. Water was allowed, as desired, except for 1 hour before and after dosing.

Subjects were not allowed to lie down for the first 4 hours after ozanimod dosing.

\section{Key Inclusion and Exclusion Criteria}

Eligible participants were women and men aged 18 through 55 years with a body weight of at least $50 \mathrm{~kg}$ and a body mass index of 18 to $30 \mathrm{~kg} / \mathrm{m}^{2}$. Subjects were required to be in good health, as determined by no clinically significant findings from medical and surgical history, physical examination, and vital signs. Subjects agreed to use adequate contraception during the study and until 30 days after receiving the last dose of study drug and to comply with all study requirements.

Excluded from participation were subjects who were pregnant or breastfeeding or who had any history or clinical manifestation of any significant endocrine, metabolic, allergic, dermatologic, hepatic, renal, hematologic, pulmonary, cardiovascular, gastrointestinal, neurologic, or psychiatric disorder. Also excluded were subjects with the presence or history of any abnormality or illness that, in the opinion of the study investigator, could affect the absorption, distribution, metabolism, or elimination of the study drug or could limit the subject's ability to participate in and complete the trial. Other exclusion criteria included significant abnormalities detected by 12-lead ECG or screening laboratory tests; history of alcoholism, drug abuse, or addiction within 24 months prior to screening; a positive serum result for the human immunodeficiency virus or hepatitis infection; use of tobacco- or nicotine-containing products within 3 months of study initiation; and participation in any other investigational study within 90 days or 5 times the half-life of the study drug, whichever was longer.

Prior to dosing and throughout the study, subjects were prohibited from using prescription or overthe-counter medications (excluding contraceptives and acetaminophen) and from consuming alcohol, grapefruits, Seville oranges, or any products containing these ingredients.

\section{Assessments}

During each treatment period, blood samples for PK analysis were collected at predose (0 hours) and $0.5,1,2,3,4,6,8,12,24,36,48$, and 72 hours postdose. Plasma concentrations of ozani- mod, RP101988, and RP101075 were determined by ICON Laboratory Services, Inc. (Whitesboro, New York) using a validated liquid chromatography-tandem mass spectrometry assay. Ozanimod, RP101988, and RP101075 were extracted from $0.2 \mathrm{~mL}$ of $\mathrm{K}_{2}$ ethylenediaminetetraacetic acid human solid-phase extraction (Evolute $25 \mathrm{mg} \mathrm{ABN}$, Biotage). Chromatographic separation was achieved on a Waters Acquity ultraperformance liquid chromatrography (UPLC) system, running a gradient composed of $0.1 \%$ formic acid in water and $0.1 \%$ formic acid in method at 0.350 $\mathrm{mL} / \mathrm{min}$ through a Waters Acquity UPLC HSS T3, 2.1 $\times 5.0 \mathrm{~mm}, 1.8-\mu \mathrm{m}$ guard column, and a $2.1 \times 100 \mathrm{~mm}$, $1.8-\mu \mathrm{m}$ analytical column. The column effluent was delivered to an API5000 mass spectrometer (AB Sciex) with electrospray interface operated in negative ionization mode. Analysis of analytes and the respective internal standard was done by selected reaction monitoring mode at unit mass resolution. Quantification was achieved using ratios of analyte peak area to internal standard peak area. Concentrations of the calibration curve standards, quality control samples, and study samples were determined by the method of weighted least-squares linear regression $\left(1 / \mathrm{x}^{2}\right)$. The bioanalytical method was validated over concentration ranges of 4.0 to $2000 \mathrm{pg} / \mathrm{mL}$ for ozanimod and RP101075 and 16.0 to $4000 \mathrm{pg} / \mathrm{mL}$ for RP101988. The interassay precision values were $\leq 7.24 \%$ for ozanimod, $\leq 7.54 \%$ for RP101988, and $\leq 10.5 \%$ for RP101075. The accuracy (\% bias) values ranged from $3.00 \%$ to $7.63 \%$ for ozanimod, $-2.50 \%$ to $0.833 \%$ for RP 101988 , and $0.833 \%$ to $6.94 \%$ for RP101075.

For the calculation of concentration summaries, all concentrations below the limit of quantification were to be treated as zero. The following PK parameters were derived by noncompartmental methods, using actual collection times and validated PK software (Phoenix WinNonlin Professional Network Edition, version 6.3.0.395; Certara, Princeton, New Jersey): AUC from time 0 extrapolated to infinity $\left(\mathrm{AUC}_{0-\infty}\right)$ or to the last quantifiable concentration $\left(\mathrm{AUC}_{0-\mathrm{t}}\right)$, maximum plasma concentration $\left(\mathrm{C}_{\max }\right), \mathrm{T}_{\max }$, terminal elimination half-life $\left(\mathrm{t}_{1 / 2}\right)$, apparent oral clearance $(\mathrm{CL} / \mathrm{F}$; ozanimod only), and apparent volume of distribution associated with terminal phase $\left(\mathrm{V}_{\mathrm{d}} / \mathrm{F}\right.$; ozanimod only).

Clinical laboratory tests, 12-lead ECGs, measurement of vital signs, and recording AEs were performed throughout the study. Use of concomitant medications was monitored throughout the study.

\section{Statistical Analysis}

A sample size of 24 subjects per treatment was estimated to provide at least $80 \%$ power to reject both null hypotheses in favor of the alternative hypothesis that the means of the primary $\mathrm{PK}$ parameters $\left(\mathrm{AUC}_{0-\infty}\right.$ and 
$\mathrm{C}_{\max }$ ) were comparable under fed and fasted conditions, assuming a 2-1-sided $t$ test significance level of 0.05 and that the intrasubject variability of the difference between treatments on the natural $\log$ scale did not exceed 0.35 for any primary PK parameter.

Primary PK parameters were analyzed in linear mixed-effects models to calculate the geometric mean ratio of each parameter in the fed state (high fat and low fat separately) relative to that in the fasted state, using natural log-transformed data. The difference in least-squares geometric means and the $90 \%$ confidence intervals (CIs) of the difference were back-transformed to provide the treatment mean ratios (test:reference) for each primary PK parameter. The test formulations were high-fat meal (treatment B) and low-fat meal (treatment $\mathrm{C}$ ), and the reference formulation was the fasted state (treatment A).

The absence of a food effect was to be declared if the $90 \%$ CI for the ratio of the population geometric means between the fed and fasted states was contained within the default limits of 0.8 to 1.25 for both $\mathrm{AUC}_{0-\infty}$ and $\mathrm{C}_{\max }$.

All statistical tabulations and analyses were performed using SAS, version 9.3 (SAS Institute, Cary, North Carolina).

\section{Results}

\section{Subjects}

Twenty-four subjects were enrolled, and all received at least 1 dose of the study drug. Hence, all enrolled subjects were evaluated for safety. Three subjects did not complete the study, 1 because of asymptomatic alanine aminotransferase $\geq 2$ times the upper limit of normal (missed treatment B in sequence ACB), 1 because of a protocol deviation (positive urine drug-screen result; missed treatment $\mathrm{A}$ in sequence CBA), and 1 because of the inability to consume the high-fat breakfast (missed treatment $\mathrm{B}$ in sequence $\mathrm{ACB}$ ). One additional subject was not dosed during period 2 (missed treatment $B$ in sequence $\mathrm{ABC}$ ) because of the inability to complete the high-fat breakfast. However, this subject continued in the study and was dosed in period 3 according to the assigned treatment sequence. Demographics and baseline characteristics of the study population are summarized in Table 1.

\section{Pharmacokinetics}

The mean plasma concentration-time profiles for ozanimod, RP101988, and RP101075 following a single 1-mg oral dose of ozanimod $\mathrm{HCl}$ were similar under fasted and fed conditions (Figure 1). The PK parameters for ozanimod, RP101988, and RP101075 are summarized in Table 2. All PK parameters for ozanimod, RP101988, and RP101075 other than $\mathrm{T}_{\max }$ were
Table I. Demographics and Baseline Characteristics of the Study Population $(\mathrm{N}=24)$

\begin{tabular}{lc}
\hline Age, mean (range), y & $34.8(18-55)$ \\
\hline Weight, mean (range), kg & $73.0(50.5-100.2)$ \\
Body mass index, mean (range), $\mathrm{kg} / \mathrm{m}^{2}$ & $25.9(20.2-29.6)$ \\
Sex, $\mathrm{n}(\%)$ & \\
Male & $10(41.7)$ \\
Female & $14(58.3)$ \\
Ethnicity, $\mathrm{n}(\%)$ & \\
Hispanic or Latino & $10(41.7)$ \\
Not Hispanic or Latino & $14(58.3)$ \\
Race, $\mathrm{n}$ (\%) & \\
Asian & $1(4.2)$ \\
Black or African American & $6(25.0)$ \\
White & $17(70.8)$ \\
\hline
\end{tabular}
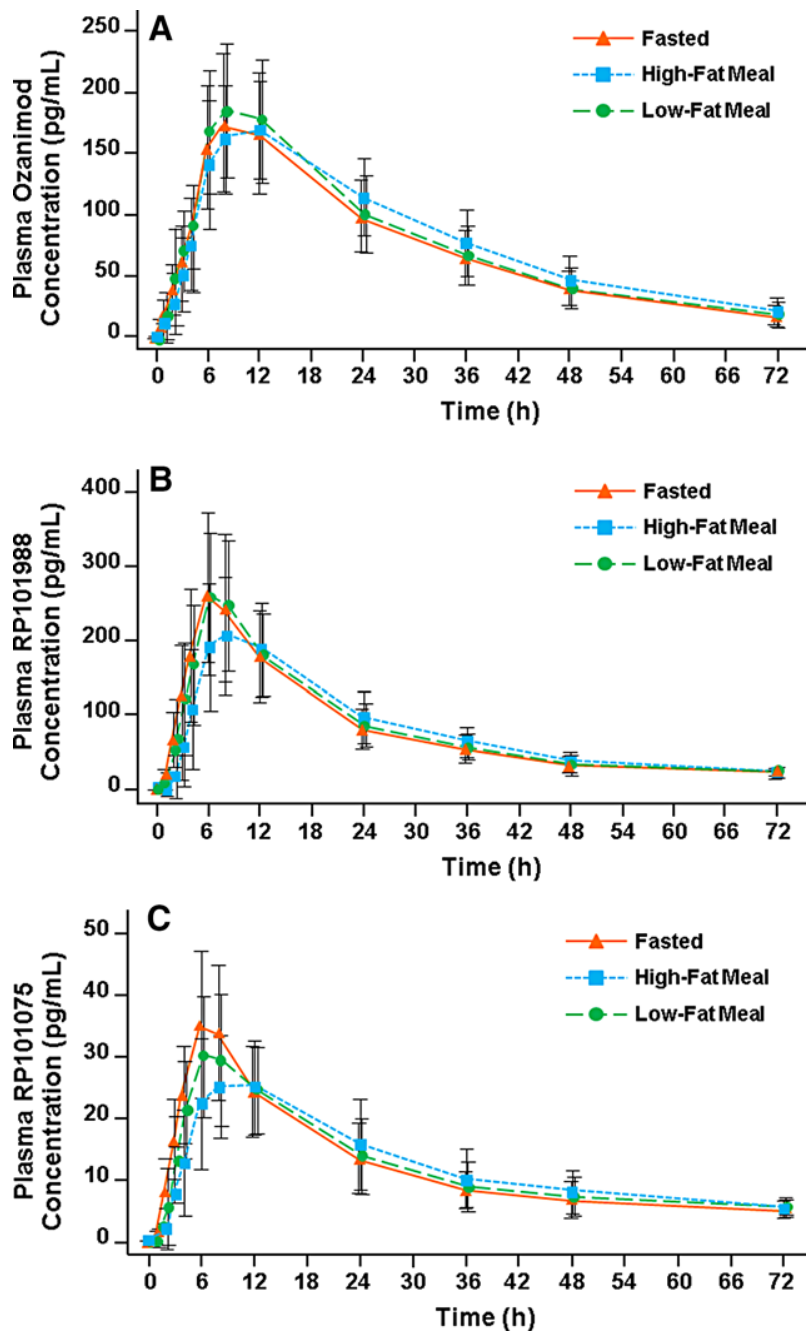

Figure I. Mean (SD) plasma concentration-time profiles for ozanimod (A), RPIOI 988 (B), and RPI0I075 (C) following a single $\mathrm{I}-\mathrm{mg}$ oral dose of ozanimod $\mathrm{HCl}$ under fasted and fed conditions. 
Table 2. Summary of Pharmacokinetic Parameters Following a Single I-mg Ozanimod $\mathrm{HCl}$ Oral Dose in Fed and Fasted States $(\mathrm{n}=2 \mathrm{I}-24)$

\begin{tabular}{|c|c|c|c|}
\hline Parameter $^{\mathrm{a}}$ & Fasted & High-Fat Breakfast & Low-Fat Breakfast \\
\hline \multicolumn{4}{|l|}{ Ozanimod } \\
\hline $\mathrm{AUC}_{0-\infty}(\mathrm{pg} \cdot \mathrm{h} / \mathrm{mL})$ & $57 \mid 4(3 \mid \%)$ & 6050 (27\%) & $5998(33 \%)$ \\
\hline $\mathrm{C}_{\max }(\mathrm{pg} / \mathrm{mL})$ & $18 \mid(31 \%)$ & $184(21 \%)$ & $192(28 \%)$ \\
\hline $\mathrm{T}_{\max }(\mathrm{h})$ & $8.00(6.00-12.00)$ & $12.00(6.00-12.00)$ & $8.00(6.00-12.00)$ \\
\hline$t_{1 / 2}(h)$ & $19.2(15 \%)$ & $19.2(15 \%)$ & $19.3(18 \%)$ \\
\hline \multicolumn{4}{|l|}{ RPI0I988 } \\
\hline $\mathrm{AUC}_{0-\infty}(\mathrm{pg} \cdot \mathrm{h} / \mathrm{mL})$ & $5960(33 \%)$ & $5782(31 \%)$ & $5823(33 \%)$ \\
\hline $\mathrm{C}_{\max }(\mathrm{pg} / \mathrm{mL})$ & $266(42 \%)$ & $223(371 \%)$ & $264(33 \%)$ \\
\hline $\mathrm{T}_{\max }(\mathrm{h})$ & $6.00(6.00-8.00)$ & $8.00(6.00-12.00)$ & $6.00(6.00-8.00)$ \\
\hline$t_{1 / 2}(h)$ & $18.2(17 \%)$ & $17.4(19 \%)$ & 17.5 (19\%) \\
\hline \multicolumn{4}{|l|}{ RPI0I075 } \\
\hline $\mathrm{AUC}_{0-\mathrm{t}}(\mathrm{pg} \cdot \mathrm{h} / \mathrm{mL})^{\mathrm{b}}$ & 703 (34\%) & $687(45 \%)$ & $699(40 \%)$ \\
\hline $\mathrm{C}_{\max }(\mathrm{pg} / \mathrm{mL})$ & $37.1(33 \%)$ & $29.8(28 \%)$ & $31.8(31 \%)$ \\
\hline $\mathrm{T}_{\max }(\mathrm{h})$ & $6.00(4.00-12.00)$ & $8.05(4.00-24.00)$ & $6.00(3.00-12.00)$ \\
\hline$t_{1 / 2}(h)$ & $17.0(27 \%)$ & $17.5(26 \%)$ & $21.0(49 \%)$ \\
\hline
\end{tabular}

$\mathrm{AUC}_{0-\infty}$, area under the plasma concentration-time curve from time 0 extrapolated to infinity; $\mathrm{AUC}_{0-\mathrm{t}}$, area under the plasma concentration-time curve from time 0 to the last quantifiable concentration; $C_{\max }$, maximum observed plasma concentration; $T_{\text {max }}$, time to reach $C_{\text {max }}$; $t_{I / 2}$, terminal elimination half-life.

a Data are presented as mean (\% coefficient of variation), except for $\mathrm{T}_{\text {max }}$, which is expressed as median (minimum-maximum).

${ }^{b} \mathrm{RPIOI075} \mathrm{AUC}_{0-\infty}$ could not be characterized for the majority of subjects (because of extrapolated $\mathrm{AUC} \mathrm{C}_{0-\infty}>20 \%, R^{2}<0.80$, or $<3$ points available for the determination of $\lambda_{z}$ ).

similar under the 3 treatment conditions. The median $\mathrm{T}_{\max }$ of ozanimod, RP101988, and RP101075 was delayed by 4,2 , and 2 hours, respectively, following a highfat meal compared with fasted and low-fat meal conditions. However, the range of $\mathrm{T}_{\max }$ values was similar for all treatments under fasted or fed conditions.

Only subjects who had evaluable PK parameters for both treatments were included in the corresponding comparison (B vs $\mathrm{A}$; $\mathrm{C}$ vs $\mathrm{A}$ ). Twenty subjects completed both treatments $\mathrm{A}$ and $\mathrm{B}$, and 23 subjects completed both treatments A and C. For ozanimod and RP101988, the $90 \% \mathrm{CIs}$ for the ratios of geometric least-squares means (fed/fasted) were within the equivalence limits of 0.80 to 1.25 for $\mathrm{AUC}_{0-\infty}$ and $\mathrm{C}_{\max }$ (Table 3). For RP101075, the $90 \%$ CIs for the ratios of geometric least-squares means (fed/fasted) were within the equivalence limits of 0.80 to 1.25 for $\mathrm{AUC}_{0-\mathrm{t}}$ and $\mathrm{C}_{\max }$ (Table 3), with 1 exception. This exception was a small reduction of $\sim 18 \%$ (ratio of 0.82 ) in the $\mathrm{C}_{\max }$ of RP101075 when ozanimod was administered with a high-fat meal as opposed to in the fasted state, with the $90 \% \mathrm{CIs}$ for the ratios of 0.76 to 0.88 .

\section{Safety}

There were no serious AEs. Nine subjects (37.5\%) reported at least 1 treatment-emergent $\mathrm{AE}$ (TEAE) during any treatment, including $5(21.7 \%)$ in the fasted state, $6(28.6 \%)$ with the high-fat meal, and $5(20.8 \%)$ with the low-fat meal. All events were of mild or moderate intensity, and most were considered by the investigator to be unrelated or unlikely to be related to the study drug. TEAEs that occurred in more than 1 subject were headache $(n=4 ; 16.7 \%)$ and constipation $(\mathrm{n}=2 ; 8.3 \%)$. Four subjects had TEAEs considered possibly related to the study drug, which included headache, asymptomatic alanine aminotransferase $\geq 2$ times the upper limit of normal (6 days after the second dose of study drug, resolved by day 22), abdominal pain, and second-degree atrioventricular block (Mobitz type 1). The atrioventricular block was asymptomatic, occurred on day 15 approximately 8 hours after the third dose following the high-fat meal, and lasted approximately 2 hours with spontaneous reversion.

\section{Discussion}

This phase 1 study was designed to evaluate whether food consumption (high fat or low fat) has an effect on the systemic exposure of ozanimod or its active metabolites. Specific mechanisms of drug-food interactions include delays in gastric emptying, changes in gastrointestinal $\mathrm{pH}$, increases in splanchnic blood flow or in the flow of bile salts, changes in GI viscosity, alterations in luminal metabolism or in systemic drug clearance, and direct physical or chemical interactions with the drug formulation (eg, changes in solubility and/or dissolution). ${ }^{11-13}$ The results of this study demonstrated an absence of a food effect on ozanimod and the 
Table 3. Statistical Analysis of the Effect of Each Breakfast on Pharmacokinetics

\begin{tabular}{|c|c|c|c|c|c|c|}
\hline \multirow[b]{2}{*}{ End Point } & \multicolumn{3}{|c|}{ High-Fat vs Fasted } & \multicolumn{3}{|c|}{ Low-Fat vs Fasted } \\
\hline & $\mathrm{n}$ & Ratio of Geometric LS Mean (Fed/Fasted) & $90 \% \mathrm{Cl}$ & $\mathrm{n}$ & Ratio of Geometric LS Mean (Fed/Fasted) & $90 \% \mathrm{Cl}$ \\
\hline \multicolumn{7}{|l|}{ Ozanimod } \\
\hline $\mathrm{AUC}_{0-\infty}$ & 19 & 1.10 & $1.05-1.15$ & 23 & 1.06 & $|.02-1.1|$ \\
\hline $\begin{array}{l}\mathrm{C}_{\max } \\
\mathrm{RPI0I988}\end{array}$ & 20 & 1.06 & $0.97-1.15$ & 23 & 1.08 & $1.02-1.14$ \\
\hline $\mathrm{AUC}_{0-\infty}$ & 17 & 1.02 & $0.96-1.08$ & 21 & 1.03 & $0.98-1.08$ \\
\hline $\begin{array}{l}\mathrm{C}_{\max } \\
\mathrm{RPI0I075}\end{array}$ & 20 & 0.87 & $0.82-0.93$ & 23 & 1.02 & $0.97-1.08$ \\
\hline$A \cup C_{0-t}$ & 20 & 0.96 & $0.87-1.05$ & 23 & 0.99 & $0.93-1.05$ \\
\hline $\mathrm{C}_{\max }$ & 20 & 0.82 & $0.76-0.88$ & 23 & 0.87 & $0.80-0.94$ \\
\hline
\end{tabular}

$\mathrm{AUC}_{0-\infty}$, area under the plasma concentration-time curve from time 0 extrapolated to infinity; $\mathrm{AUC}_{0-\mathrm{t}}$, area under the plasma concentration-time curve from time 0 to the last quantifiable concentration; $\mathrm{Cl}$, confidence interval; $\mathrm{C}_{\max }$, maximum observed plasma concentration; $\mathrm{LS}$, least squares.

major active metabolite RP101988. A food effect is considered demonstrable if the $90 \% \mathrm{CI}$ for the ratio of population geometric means between fed and fasted treatment for $\mathrm{C}_{\max }$ and $\mathrm{AUC}$ does not meet the $80 \%$ to $125 \%$ bioequivalence criterion. ${ }^{13}$ Although an absence of the low-fat food effect on the minor active metabolite RP101075 was established, it was not demonstrated for the high-fat food effect because the lower bound of the $90 \% \mathrm{CI}$ for the ratio of geometric least-squares means was outside the no-effect boundary of 0.80 to 1.25 for the RP101075 $\mathrm{C}_{\max }$. However, this minor change is not considered clinically meaningful because the extent of exposure $\left(\mathrm{AUC}_{0-t}\right)$ of RP101075 was unaffected, and the lower $\mathrm{C}_{\max }$ of this minor active metabolite was not expected to have any effect on clinical safety or efficacy.

Prior to the availability of the food-effect study results, patients in the ozanimod phase 2 studies in RMS and UC were instructed to take ozanimod in fasting conditions. Results of the ozanimod phase 2 studies in RMS and UC showed clinical benefit, along with an acceptable safety profile, for the ozanimod $\mathrm{HCl} 0.5$ - and 1-mg once-daily regimens. ${ }^{14,15}$ In RADIANCE Part-A, a phase 2 randomized, placebo-controlled trial among 258 patients with RMS, the number of gadoliniumenhancing lesions in weeks 12 through 24 (primary end point) was significantly lower for patients on oncedaily doses of ozanimod $\mathrm{HCl} 0.5$ or $1 \mathrm{mg}$ than for placebo recipients. The mean cumulative number of total gadolinium-enhancing lesions was 1.5 (standard deviation, 3.7) with ozanimod $\mathrm{HCl} 0.5 \mathrm{mg}$ and 1.5 (3.4) with ozanimod $1 \mathrm{mg}$ compared with 11.1 (29.9) for placebo $\left(P<.0001\right.$ vs placebo for both doses) ${ }^{14}$ Both doses of ozanimod also were associated with a significantly lower mean number of gadolinium-enhancing lesions in week 24 ( $P<.0001$ vs placebo for both doses) and with significant reductions in the mean number of T2 lesions from weeks 12 through $24(P<.0001$ vs placebo for both doses). ${ }^{14}$ Treatment was very well tol- erated, with no study discontinuations attributable to an AE. The most common AEs (nasopharyngitis and headache) were reported more frequently by patients who received placebo. ${ }^{14}$ In the phase 2 double-blind, placebo-controlled TOUCHSTONE trial, 197 adults with UC were randomized to receive at least 1 dose of ozanimod $\mathrm{HCl}(0.5$ or $1 \mathrm{mg})$ or placebo (1:1:1) once daily for up to 32 weeks. ${ }^{15}$ By week 8 , significantly more patients receiving ozanimod $\mathrm{HCl} 1 \mathrm{mg}$ (vs placebo) had achieved clinical remission, the primary end point $(16 \%$ vs $6 \% ; P=.048)$, whereas the difference between ozanimod $\mathrm{HCl} 0.5 \mathrm{mg}$ and placebo (14\% vs $6 \% ; P=0.14)$ was not statistically significant. In exploratory analyses, both doses of ozanimod were associated with greater clinical remission, clinical response, mucosal healing, and histologic remission versus placebo in week 32 (differences were nominal and not significant). ${ }^{15}$ There were no evident differences between groups for the most commonly reported AEs (anemia and headache).

In conclusion, results from this study show that food intake did not have an effect on exposure of ozanimod or its active metabolites. Therefore, ozanimod can be taken without regard to meals.

\section{Declaration of Conflicting Interests}

J.Q. Tran is a current employee of Receptos and is a stockholder in Celgene Corporation. At the time of the study and analysis, J.P. Hartung, C. Tompkins, and P.A. Frohna were employees of Receptos. J.P. Hartung is currently employed by JPH Clinical Development, Inc. (San Diego, California). P.A. Frohna is currently employed by Bioniz Therapeutics, Inc. (Irvine, California). The authors received writing and editorial support for article preparation from Susan Martin, $\mathrm{PhD}$, and Philip Sjostedt, BPharm, MPH, from the Medicine Group, which was paid for by Receptos. The authors, however, directed and are fully responsible for all content and editorial decisions for this article. 


\section{Funding}

This study was supported by Receptos, a wholly owned subsidiary of Celgene Corporation.

\section{References}

1. Tullman MJ. A review of current and emerging therapeutic strategies in multiple sclerosis. Am J Manag Care. 2013;19(2 Suppl):S21-27.

2. Ng SC, Kamm MA. Therapeutic strategies for the management of ulcerative colitis. Inflamm Bowel Dis. 2009;15(6):935-950.

3. Lugaresi A. Addressing the need for increased adherence to multiple sclerosis therapy: can delivery technology enhance patient motivation? Expert Opin Drug Deliv. 2009;6(9):995-1002.

4. Shah ED, Siegel CA, Chong K, Melmed GY. Patients with Crohn's disease are more likely to remain on biologics than immunomodulators: a meta-analysis of treatment durability. Dig Dis Sci. 2015;60(8):2408-2418.

5. Subei A, Cohen J. Sphingosine 1-phosphate receptor modulators in multiple sclerosis. CNS Drugs. 2015;29(7):565-575.

6. Juif PE, Kraehenbuehl S, Dingemanse J. Clinical pharmacology, efficacy, and safety aspects of sphingosine-1phosphate receptor modulators. Expert Opin Drug Metab Toxicol. 2016;12(8):879-895.

7. Strub GM, Maceyka M, Hait NC, Milstien S, Spiegel S. Extracellular and intracellular actions of sphingosine-1phosphate. Adv Exp Med Biol. 2010;688:141-155.
8. Sorensen PS. Ozanimod: a better or just another S1P receptor modulator? Lancet Neurol. 2016;15(4):345347.

9. Scott FL, Clemons B, Brooks J, et al. Ozanimod (RPC1063) is a potent sphingosine-1-phosphate receptor-1 (S1P1) and receptor-5 (S1P5) agonist with autoimmune disease-modifying activity. Br J Pharmacol. 2016;173(11):1778-1792.

10. Tran JQ, Hartung JP, Peach RJ, et al. Results from the first-in-human study with ozanimod, a novel, selective sphingosine-1-phosphate receptor modulator. J Clin Pharmacol. 2017;57(8):988-996.

11. Singh BN. Effects of food on clinical pharmacokinetics. Clin Pharmacokinet. 1999;37(3):213-255.

12. Lentz KA. Current methods for predicting human food effect. AAPS J. 2008;10(2):282-288.

13. Food and Drug Administration. Guidance for industry - Food-effect bioavailability and fed bioequivalence studies. 2002. http://www.fda.gov/downloads/Regulat oryInformation/Guidances/UCM126833.pdf. Accessed February 1, 2017.

14. Cohen JA, Arnold DL, Comi G, et al. Safety and efficacy of the selective sphingosine1-phosphate receptor modulator ozanimod in relapsing multiple sclerosis (RADIANCE): a randomised, placebo-controlled, phase 2 trial. Lancet Neurol. 2016;15(4):373-381.

15. Sandborn WJ, Feagan BG, Wolf DC, et al. Ozanimod induction and maintenance treatment for ulcerative colitis. $N$ Engl J Med. 2016;374(18):1754 1762. 